\title{
CRITICAL CONTACT RATE FOR VECTOR- HOST-PATHOGEN OSCILLATION INVOLVING CO-FEEDING AND DIAPAUSE
}

\author{
XUE ZHANG* \\ Department of Mathematics, Northeastern University \\ Shenyang, Liaoning, 110819, P. R. China \\ *zhangxue@mail.neu.edu.cn \\ XIAOTIAN WU ${ }^{\dagger}$ \\ Department of Mathematics, Shanghai Maritime University \\ Shanghai, 201306, P. R. China \\ ${ }^{\dagger} x t w u @ s h m t u . e d u . c n$ \\ JIANHONG WU \\ Laboratory for Industrial and Applied Mathematics \\ York University, Toronto, ON, M3J1P3, Canada \\ wujh@yorku.ca
}

Received 1 January 2017

Revised 8 September 2017

Accepted 14 October 2017

Published 1 December 2017

\begin{abstract}
We consider the dynamic vector-host-pathogen interaction motivated by tick-borne diseases such as tick-borne encephalitis and Lyme disease. We stratify the vector population in terms of the stage before and after the vector's contact with hosts when co-feeding transmission may take place, and we also consider the case where vector development may involve two time lags due to normal development and diapause. We derive threshold conditions for disease persistence and for nonlinear oscillations in the vector population and in the diseased vector and host populations. Our objective here is to use a simple mechanistic dynamic model to show that diapause and co-feeding transmission may generate periodic and irregular oscillations even when seasonal variations of the environmental conditions are ignored. These oscillations are not necessary in synchrony with the seasonality of vector development, and hence complicated oscillatory patterns of vectorborne disease dynamics in the field and surveillance observations should be expected.
\end{abstract}

Keywords: Diapause; Tick Population Dynamics; Tick-Borne Disease; Oscillation.

\section{Introduction}

We consider the transmission dynamics of tick-borne diseases such as tick-borne encecphalitis and Lyme disease. Tick-borne diseases can be caused by infection

${ }^{*}{ }^{\dagger}$ Corresponding authors.

This is an Open Access article published by World Scientific Publishing Company. It is distributed under the terms of the Creative Commons Attribution 4.0 (CC-BY) License. Further distribution of this work is permitted, provided the original work is properly cited. 
with a variety of pathogens, including rickettsia and other types of bacteria, viruses and protozoa. In Europe and Asia, Ixodes ricinus and Ixodes persulcatus ticks are primary species transmitting tick-borne encephalitis and Lyme borreliosis, although infections can also be transmitted through ingesting contaminated unpasteurized cow, sheep or goat milk and milk products obtained from infected animals. Ticks can be found primarily in forests, wooded parks, grasslands and low-growing dense bush, they normally have three post-egg stages: larva, nymph and adult! 15

Mathematical models have been developed to estimate tick-host interaction and pathogen transmission dynamics. Rosà and Pugliese ${ }^{6}$ assessed how changes in host species densities can influence the persistence of tick-borne pathogen. Their work considered different infection routes occurring between hosts and ticks. Dunn et al! ${ }^{7}$ performed a global sensitivity analysis to rank the importance of model parameters in terms of their contribution to the observed variations in the basic reproduction number. They concluded that there are three most influential factors: the transmission efficiency from the vertebrate host to ticks, the survival rate from fed larva to feeding nymph, and the fraction of nymphs finding a competent host. Lou et al ${ }^{8}$ proposed a stage-structured periodic model to identify the tick reproduction threshold and the disease spread threshold, and investigated effects of seasonal temperature variation and host community composition on the pattern of Lyme disease spread. Unfortunately, none of these models have incorporated the roles of co-feeding transmission and diapause.

The tick's life cycle is at least one year and normally lasts $2-3$ years. This creates a long delay in the population dynamics model of ticks at a particular stage. In addition, adverse environmental conditions or a decline in day length may cause ticks to enter diapause, leading to further delay in seeking, development, or oviposition for a year or longer. Furthermore, a post-egg tick must take a blood meal from an appropriate host to progress from one stage to another. In each progress, a tick successfully quested a host attaches and bites the host, and remains in the host until engorging, and then subsequently developing into the next stage ${ }^{[}$During this progress, co-feeding transmission can take place when one susceptible tick may get infection by co-feeding with another infected tick (normally at a later stage) in the same host. Our focus here is to formulate a simple model to describe the roles of diapause and co-feeding transmission in tick population dynamics and tickborne pathogen spread. A minimal set of compartments in such a model must involve tick populations in two different stages (relevant to the co-feeding) and two different development lags (one is related to the diapause effect). A natural question is whether such a model can exhibit nonlinear oscillations.

\section{The Framework and Hypotheses}

We consider a system consisting of a bacteria pathogen, a vector population with two stages, and a host population which is considered as a vehicle capable of transmitting the pathogen through both co-feeding and systemic transmission routes. We now list our major assumptions. 


\section{Model Assumptions:}

(i) In terms of the bacteria pathogen, the host and vector populations are stratified by their infection status as susceptible $(s)$ and infected (i);

(ii) We assume, in reference to the co-feeding process (described below), the vector population is divided into two distinguished subgroups, the subgroup of ticks questing for the first (post-egg stage) feeding (B) and the subgroup of ticks questing for the second feeding (A). The feedback from A to B can be the outcome of a sequence of developments and reproduction irrelevant to the co-feeding process. Note also that we have implicitly ignored the immediate feeding and engorging process for a B-stage questing tick to an A-stage questing tick, very much like the normal practice of SIR modelling when the incubation and/or latent period is omitted.

(iii) Once infected vectors in the A-subgroup co-feed on a host with susceptible vectors in the B-subgroup, co-feeding may take place so that the B-stage susceptible vectors can be infected due to the co-feeding. Note that if the host is already infected, the B-stage susceptible vectors can also be infected through the systemic infection route.

(iv) A susceptible host can be infected if it is bitten by an infected vector (at either stage) and this infection rate can be described by the mass action;

(v) In addition to subcategory for being susceptible or infected, a host is further divided into being bitten (infested) or not by some infected A-stage vectors to reflect if co-feeding is possible. So we have $H_{s^{+}}, H_{s^{-}}, H_{i^{+}}, H_{i^{-}}$, here $+/-$ indicating that the host is/is not infested by A-stage infected ticks for the realization of co-feeding transmission. More precisely, $H_{s^{+}}$is the subgroup of hosts which are currently susceptible to the infection, currently fed (infested) by infected vectors at the A-stage, and can pass infection to the co-feeding B-stage ticks. In contrast, $H_{s^{-}}$is the subgroup of hosts which are currently susceptible to the infection, with a small number of (or no) infected vectors at the A-stage infested, but cannot pass infection to the co-feeding B-stage ticks. Throughout this work, co-feeding transmission refers to the transmission to a susceptible B-stage vector from some infected A-stage vectors when they are co-feeding on the same host, while systemic infection refers to a susceptible vector gets infection through bites from an infected host. Whether co-feeding transmission takes place or not depends on a large number of factors including the co-feeding duration and the density and (physical) proximity of A-stage infected ticks to the susceptible B-stage ticks. 10,11 Here, we made a simplified assumption that there are only two groups of hosts: those in which co-feeding can take place and those co-feeding cannot happen (note that this is different from the assumption whether the host has one or none infested tick). A more realistic model formulation will have to involve the density and spatial distributions of the infected A-stage ticks and their relative spatial locations to the B-stage susceptible ticks within the same host. We remark that $H_{i^{+}}$is the 


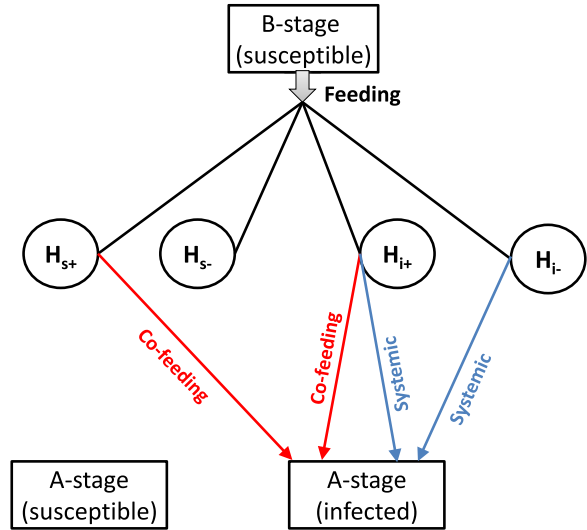

(a)

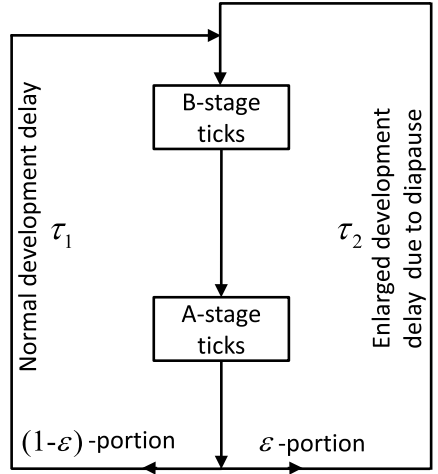

(b)

Fig. 1. Illustrations show (a) co-feeding and systemic transmissions and (b) delays induced by normal development and diapause. At (a), a B-stage vector can get infection by feeding (i) a host from the subgroup $H_{s}$ through co-feeding transmission route; (ii) a host from the subgroup $H_{i-}$ through the systemic transmission route; and (iii) a host from the subgroup $H_{i+}$ through both co-feeding and systemic transmission routes. Development from a B-stage vector to a susceptible A-stage host is not marked in the illustration. At (b), a portion of A-stage ticks produces the next generation of B-stage vectors with an enlarged delay $\tau_{2}$ than the normal development delay $\tau_{1}$ for ticks without experiencing the diapause.

subgroup of hosts which are already infected and are also currently infested by the A-stage infected vectors, so a susceptible B-stage vector can have the infection risk from both systemic and co-feeding transmission routes if this B-stage vectors co-feed on the same host in the host group $H_{i^{+}}$(see Fig. 11(a) for an illustration).

(vi) Normally, it takes a development delay (denoted by $\tau_{1}$ ) for a A-stage tick to complete a sequence of developments, reproduction (into eggs) and a further development (molting) to generate some B-stage vectors. Due to diapause, 12 14 a portion $(\epsilon)$ of these A-stage ticks will complete this sequence with a larger development delay $\tau_{2}$, with $\tau_{2}>\tau_{1}$ (see Fig. [1(b) for an illustration).

\section{The Model}

We now introduce model variables relevant to our particular focus on diapause and co-feeding. We will use $T_{B}(t)$ and $T_{A}(t)$ to denote the number of ticks questing for the first (post-egg) and second feeding, respectively, and $H(t)$ the total number of hosts involved in the transmission from B-stage to A-stage of ticks. In terms of the status of infection, $T_{A s}(t)$ and $T_{A i}(t)$ represent the numbers of susceptible and infected A-stage ticks at time $t$, and $T_{A}(t)=T_{A s}(t)+T_{A i}(t)$ is the total number of A-stage ticks.

The dynamics of $T_{B}(t)$, following Fig. [1(b), is described by

$$
T_{B}^{\prime}(t)=\rho_{1} b\left(\rho_{2}\left[(1-\epsilon) T_{A}\left(t-\tau_{1}\right)+\epsilon T_{A}\left(t-\tau_{2}\right)\right]\right)-C_{B} H T_{B}-d_{B} T_{B},
$$


where $\rho_{1}$ is the survival rate from eggs to B-stage, $b(x)$ is the egg production function, $\rho_{2}$ is the survival rate from the A-stage to egg-laying stage, $\tau_{2}>\tau_{1}$ are the development delays from A-stage to the B-stage of ticks with or without diapause, and we assume $\epsilon$-portion of A-stage ticks experience the diapause; $d_{B}$ is the death rate of ticks at B-stage. In the above formulation, it is assumed that each B-stage vector is making $C_{B}$-number of effective contacts (attachment followed by feeding) with a host. In our analysis below, the Ricker function is used for the reproduction function, that is,

$$
b(x)=r_{T} x e^{-s_{T} x}, \quad x \geq 0,
$$

where $r_{T}$ is the maximal number of eggs that an egg-laying female can lay per unit time since $b^{\prime}(0)=r_{T}$, and $s_{T}$ measures the strength of density dependence. This assumption reflects the ecological consideration that "the reproduction is linear in $x$ only for small densities, decreases as a consequence of intra specific competition, and then drops significantly at very large densities due to the available resources being utilized by the adults only for their own physiological maintenance" 15 We note that

$$
b(x) \leq b_{\infty}:=b\left(s_{T}^{-1}\right)=r_{T}\left(s_{T} e\right)^{-1} \text { for all } x \geq 0 .
$$

It is more complicated to describe the dynamics of $T_{A}(t)$ due to co-feeding and systemic transmissions. First, we use $m_{B}$ to denote the molting proportion of Bstage ticks after the first feeding, so $m_{B} C_{B} T_{B}(t) H(t)$ is the total amount of inflow into A-stage ticks. Then, A-stage ticks seek hosts again and attach them for feeding, i.e., the corresponding feeding rate can be given by the product $C_{A} T_{A}(t) H(t)$, where $C_{A}$ represents contact rate between hosts and A-stage ticks. This yields

$$
T_{A}^{\prime}(t)=m_{B} C_{B} T_{B}(t) H(t)-d_{A} T_{A}(t)-C_{A} T_{A}(t) H(t),
$$

where $d_{A}$ is the tick death rate at A-stage. We use the classical logistic model for host population dynamics:

$$
H^{\prime}(t)=r_{h} H(t)\left(1-\frac{H(t)}{K_{h}}\right)-d_{h} H(t),
$$

where $d_{h}$ is the death rate per host, $r_{h}$ is the intrinsic growth rate and $K_{h}$ the carrying capacity of hosts. Then, we end up with the following vector-host population dynamics system

$$
\left\{\begin{array}{l}
T_{B}^{\prime}(t)=\rho_{1} b\left(\rho_{2}\left[(1-\epsilon) T_{A}\left(t-\tau_{1}\right)+\epsilon T_{A}\left(t-\tau_{2}\right)\right]\right)-C_{B} H(t) T_{B}-d_{B} T_{B}, \\
T_{A}^{\prime}(t)=m_{B} C_{B} H(t) T_{B}-C_{A} T_{A} H(t)-d_{A} T_{A}, \\
H^{\prime}(t)=r_{h} H(t)\left(1-\frac{H(t)}{K_{h}}\right)-d_{h} H .
\end{array}\right.
$$

To describe the epidemiological dynamics for ticks, we use $\eta_{s}$ to denote the proportion at which an A-stage vector newly developed from a successfully molted B-stage vector fed on an infected host becomes infected through the systemic 
transmission. Therefore, $\eta_{s} m_{B} C_{B} H_{i-} T_{B}(t)$ gives the total inflow into $T_{A i}(t)$ due to the contacts of B-stage vectors fed on $H_{i^{-}}$hosts. We also make a simplified assumption about the co-feeding transmission that the hosts involved for the vectors transition from B-stage to A-stage have only two categories; those infested with infected A-stage vectors which can transmit the disease to susceptible B-stage vectors and those without or with lower densities of infected A-stage vectors which cannot transmit the disease to susceptible B-stage vectors through the co-feeding transmission route. We assume that an A-stage vector newly developed from a successfully molted B-stage vector fed on a $H_{s^{+}}$or $H_{i^{+}}$host can get the infection through the co-feeding transmission route. We use $\eta_{c}$ to denote the proportion that a B-stage vector get infection from co-feeding infected A-stage ticks. For instance, the total inflow into $T_{A i}(t)$ due to $T_{B}(t)$ contacting with $H_{s^{+}}$is $\eta_{c} m_{B} C_{B} H_{s^{+}}(t) T_{B}(t)$. These detailed systemic and co-feeding transmissions are illustrated in Figs. 2(a) and 2 (b), thereby the dynamics of $T_{A s}(t)$ and $T_{A i}(t)$ are given by

$$
\begin{aligned}
T_{A s}^{\prime}(t)= & \left(1-\eta_{c}\right) m_{B} C_{B} H_{s^{+}} T_{B}+m_{B} C_{B} H_{s^{-}} T_{B}+\left(1-\eta_{s}\right) m_{B} C_{B} H_{i^{-}} T_{B} \\
& +\left(1-\eta_{s}\right)\left(1-\eta_{c}\right) m_{B} C_{B} H_{i^{+}} T_{B}-C_{A} T_{A s} H-d_{A} T_{A s}, \\
T_{A i}^{\prime}(t)= & \eta_{c} m_{B} C_{B} H_{s^{+}} T_{B}+\left(\eta_{s}+\eta_{c}-\eta_{s} \eta_{c}\right) m_{B} C_{B} H_{i^{+}} T_{B} \\
& +\eta_{s} m_{B} C_{B} H_{i^{-}} T_{B}-C_{A} T_{A i} H-d_{A} T_{A i},
\end{aligned}
$$

where $\eta_{s}+\eta_{c}-\eta_{c} \eta_{s}$ is the proportion that the tick gets systemic infection $\left(\eta_{s}\right)$ and additional co-feeding infection $\left(\eta_{c}\left(1-\eta_{s}\right)\right)$, or alternatively, $\eta_{s}+\eta_{c}-\eta_{c} \eta_{s}$ is the proportion that the tick gets co-feeding infection $\left(\eta_{c}\right)$ and additional systemic infection $\left(\eta_{s}\left(1-\eta_{c}\right)\right)$.

For the host dynamics, as we mentioned above, the classical logistic model is used to model the total host population (Eq. (3.5)). To model $H_{s^{+}}, H_{s^{-}}, H_{i^{+}}$and $H_{i^{-}}$in detail, we denote by $d_{f}$ the rate at which a $H_{s^{+}}\left(\right.$or $\left.H_{i^{+}}\right)$host becomes $H_{s^{-}}$(or $H_{i^{-}}$respectively) due to ticks dropping off the host, and $C_{A}$ be the contact rate between an A-stage vector and a host (so that after the contact, a host

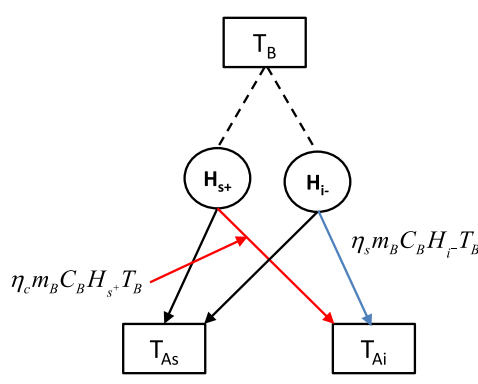

(a)

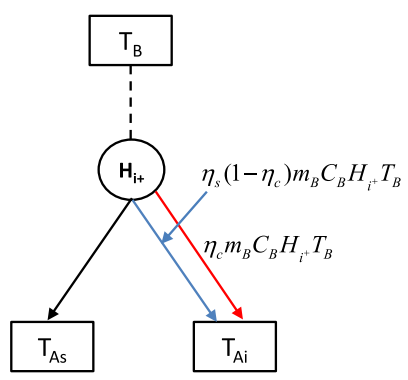

(b)

Fig. 2. Illustrations show co-feeding and systemic transmission when $T_{B}$ vectors feed on (a) $H_{s^{+}}$ and $H_{i-}$ hosts, and (b) $H_{i^{+}}$, respectively. 
Table 1. Model parameters description.

\begin{tabular}{ll}
\hline Parameter & \multicolumn{1}{c}{ Description } \\
\hline$\rho_{1}$ & Survival rate of eggs developing into B-stage ticks \\
$\rho_{2}$ & Survival rate of A-stage ticks becoming egg-laying adult female \\
$r_{T}$ & Maximum number of eggs produced by an egg-laying adult female \\
$s_{T}$ & Reproduction reduction of egg-laying adult females \\
$\tau_{1}$ & Normal time delay of A-stage ticks developing into egg-laying adults \\
$\tau_{2}$ & Enlarged time delay of A-stage ticks developing into egg-laying adults \\
& due to diapause \\
$\epsilon$ & Proportion of ticks experiencing diapause \\
$C_{B}$ & Contact rate between hosts and B-stage ticks \\
$d_{B}$ & Death rate of B-stage ticks \\
$d_{A}$ & Death rate of A-stage questing ticks \\
$m_{B}$ & Molting proportion of B-stage after the first feeding \\
$\eta_{c}$ & Proportion that susceptible ticks get infection from co-feeding transmission \\
$\eta_{s}$ & Proportion of susceptible ticks get infection through systemic transmission \\
$C_{A}$ & Contact rate between hosts and A-stage ticks, including the proportion \\
& of infectious ticks that are free to bound to a host \\
$\eta_{h}$ & Proportion of uninfected hosts become infected when bitten by infected ticks \\
$d_{f}$ & Rate at which an A-stage tick leaves $H_{.+}$for $H .-$ \\
$d_{h}$ & Death rate of hosts \\
$r_{h}$ & Intrinsic growth rate of hosts \\
$K_{h}$ & Carrying capacity of hosts \\
\hline
\end{tabular}

in the category "-" moves to the category "+"). Thereby, the host dynamics is described by

$$
\begin{aligned}
& H_{s^{+}}^{\prime}(t)=-\eta_{h} C_{A} H_{s^{+}} T_{A i}+\left(1-\eta_{h}\right) C_{A} H_{s^{-}} T_{A i}-d_{f} H_{s^{+}}-d_{h} H_{s^{+}}, \\
& H_{s^{-}}^{\prime}(t)=r_{h} H\left(1-\frac{H}{K_{h}}\right)-C_{A} H_{s^{-}} T_{A i}+d_{f} H_{s^{+}}-d_{h} H_{s^{-}}, \\
& H_{i^{+}}^{\prime}(t)=\eta_{h} C_{A} H_{s^{+}} T_{A i}+\eta_{h} C_{A} H_{s^{-}} T_{A i}+C_{A} H_{i^{-}} T_{A i}-d_{f} H_{i^{+}}-d_{h} H_{i^{+}}, \\
& H_{i^{-}}^{\prime}(t)=-C_{A} H_{i^{-}} T_{A i}+d_{f} H_{i^{+}}-d_{h} H_{i^{-}},
\end{aligned}
$$

where $H$ is the total number of hosts given by

$$
H(t)=H_{s^{+}}(t)+H_{s^{-}}(t)+H_{i^{+}}(t)+H_{i^{-}}(t) .
$$

Accordingly, Eqs. (3.1)-3.8 for $\left(T_{B}, T_{A s}, T_{A i}, H_{s^{+}}, H_{s^{-}}, H_{i^{+}}, H_{i^{-}}\right)$give a closed system of the vector-host dynamics. Detail explanations for model parameters are provided in Table 1

\section{Critical Value for Vector Population Oscillations}

We now consider the three-dimension model (3.6) for the vector population dynamics, where

$$
\begin{aligned}
& \rho_{1} b\left(\rho_{2}\left[(1-\epsilon) T_{A}\left(t-\tau_{1}\right)+\epsilon T_{A}\left(t-\tau_{2}\right)\right]\right) \\
& \quad=\alpha_{0}\left[(1-\epsilon) T_{A}\left(t-\tau_{1}\right)+\epsilon T_{A}\left(t-\tau_{2}\right)\right] e^{-\alpha_{1}\left[(1-\epsilon) T_{A}\left(t-\tau_{1}\right)+\epsilon T_{A}\left(t-\tau_{2}\right)\right]}
\end{aligned}
$$


with $\alpha_{0}=\rho_{1} r_{T} \rho_{2}$ and $\alpha_{1}=s_{T} \rho_{2}$. Assume

$$
R_{h}:=r_{h} d_{h}^{-1}>1
$$

and $H(0)>0$. Then, we have $H(t) \rightarrow H^{*}$ as $t \rightarrow \infty$, where

$$
H^{*}:=K_{h}\left(1-R_{h}^{-1}\right)>0 .
$$

Then, we consider the following limiting system

$$
\left\{\begin{array}{l}
T_{B}^{\prime}(t)=\rho_{1} b\left(\rho_{2} T_{A}^{\epsilon, t}\right)-\left(C_{B} H^{*}+d_{B}\right) T_{B}, \\
T_{A}^{\prime}(t)=m_{B} C_{B} H^{*} T_{B}-\left(d_{A}+C_{A} H^{*}\right) T_{A},
\end{array}\right.
$$

with

$$
T_{A}^{\epsilon, t}=(1-\epsilon) T_{A}\left(t-\tau_{1}\right)+\epsilon T_{A}\left(t-\tau_{2}\right) .
$$

From the definition of $b_{\infty}$ in (3.3), we have

$$
\lim \sup _{t \rightarrow \infty} T_{B}(t) \leq \frac{\rho_{1} b_{\infty}}{C_{B} H^{*}+d_{B}}:=T_{B}^{\infty},
$$

and

$$
\lim \sup _{t \rightarrow \infty} T_{A}(t) \leq \frac{m_{B} C_{B} H^{*} \rho_{1} b_{\infty}}{\left(d_{A}+C_{A} H^{*}\right)\left(C_{B} H^{*}+d_{B}\right)}:=T_{A}^{\infty} .
$$

Define the phase-space for (4.3) as $X:=\mathbb{R} \times C\left(\left[-\tau_{2}, 0\right], \mathbb{R}^{2}\right)\left(\right.$ recall $\left.\tau_{2} \geq \tau_{1} \geq 0\right)$ and $X^{+}:=[0,+\infty) \times C\left(\left[-\tau_{2}, 0\right], \mathbb{R}_{+}^{2}\right)$ and assume the initial data $\left(T_{B}(0), T_{A}(\cdot)\right) \in$ $X^{+}$is given, then we obtain a solution of (4.3) for all positive time $t \geq 0$ and a corresponding semiflow on $X^{+}$. The semiflow has a trivial equilibrium since $b(0)=$ 0 . The other nontrivial equilibrium is given by

$$
\left\{\begin{array}{l}
\rho_{1} b\left(\rho_{2} T_{A}^{*}\right)=\left(C_{B} H^{*}+d_{B}\right) T_{B}^{*}, \\
m_{B} C_{B} H^{*} T_{B}^{*}=\left(d_{A}+C_{A} H^{*}\right) T_{A}^{*} .
\end{array}\right.
$$

In particular, we have

$$
\rho_{1} b\left(\rho_{2} T_{A}^{*}\right)=\frac{\left(C_{B} H^{*}+d_{B}\right)\left(d_{A}+C_{A} H^{*}\right)}{m_{B} C_{B} H^{*}} T_{A}^{*} .
$$

Therefore, if we assume that the intrinsic birth rate

$$
b^{\prime}(0)=r_{T}>\frac{\left(C_{B} H^{*}+d_{B}\right)\left(d_{A}+C_{A} H^{*}\right)}{\rho_{1} \rho_{2} m_{B} C_{B} H^{*}}
$$

is satisfied, then (4.8) always has a positive solution $T_{A}^{*}>0$.

Define

$$
R_{T}:=\frac{r_{T} \rho_{1} \rho_{2} m_{B} C_{B} H^{*}}{\left(C_{B} H^{*}+d_{B}\right)\left(d_{A}+C_{A} H^{*}\right)} .
$$

$R_{T}$ is the basic reproduction number of the tick population: starting from a single tick at the B-stage (larva), the surviving probability during the B-stage to advance to A-stage is $C_{B} H^{*} /\left(C_{B} H^{*}+d_{B}\right)$ multiplying the successful molting proportion 
$m_{B}$, the survived and molted questing tick grows into the egg-laying tick after $1 /\left(d_{A}+C_{A} H^{*}\right)$ with the survival probability $\rho_{2}$, produces a total number $r_{T}$ of eggs and then grows into ticks at the B-stage with the probability $\rho_{1}$.

In what follows, we assume $R_{T}>1$. Therefore, (4.3) has one and only one positive solution $T_{A}^{*}>0$. Using (3.2), we obtain

$$
\left\{\begin{array}{l}
T_{A}^{*}=\left(s_{T} \rho_{2}\right)^{-1} \ln \left(R_{T}\right), \\
T_{B}^{*}=\left(d_{A}+C_{A} H^{*}\right)\left(m_{B} C_{B} H^{*}\right)^{-1} T_{A}^{*} .
\end{array}\right.
$$

Expression (4.11) has clear ecological interpretations: As $b^{\prime}(x)=r_{T}(1-$ $\left.s_{T} x\right) e^{-s_{T} x}=0$ if and only if $x^{*}=s_{T}^{-1}$, the maximal population size at which the birth rate is no longer increasing is $s_{T}^{-1}$ so the equilibrium value for $T_{A}^{*}$ is this maximum population size multiplied by the intrinsic growth rate $\ln R_{T}$ scaled appropriately by the survival probability of A-stage ticks to become egg-laying ticks.

Note that $b^{\prime}(x)>0$ for $x \in\left(0, s_{T}^{-1}\right)$ and $b^{\prime}(x)<0$ for $x \in\left(s_{T}^{-1},+\infty\right)$. Moreover, $b^{\prime \prime}(x)<0$ when $x \in\left(0,2 s_{T}^{-1}\right)$ and $b^{\prime \prime}(x)>0$ when $x>2 s_{T}^{-1}$.

Note also that under the condition $R_{T}>1, T_{A}^{*}>0$ is satisfied. If

$$
s_{T}^{-1} \ln \left(R_{T}\right)=: \rho_{2} T_{A}^{*}<x^{*}:=s_{T}^{-1}
$$

holds, then at the equilibrium $\left(T_{B}^{*}, T_{A}^{*}\right)$ the linearized system of (4.3) is given by

$$
\left\{\begin{aligned}
T_{B}^{\prime}(t)= & \rho_{1} \rho_{2} b^{\prime}\left(\rho_{2} T_{A}^{*}\right)\left[(1-\epsilon) T_{A}\left(t-\tau_{1}\right)+\epsilon T_{A}\left(t-\tau_{2}\right)\right] \\
& -\left(C_{B} H^{*}+d_{B}\right) T_{B}(t) \\
T_{A}^{\prime}(t)= & m_{B} C_{B} H^{*} T_{B}(t)-\left(d_{A}+C_{A} H^{*}\right) T_{A}(t) .
\end{aligned}\right.
$$

This is a positive feedback system since $\rho_{1} \rho_{2} b^{\prime}\left(\rho_{2} T_{A}^{*}\right)>0$, so the monotone dynamical theory can be applied. Under the assumption (4.12), we have

$$
\rho_{1} \rho_{2} b^{\prime}\left(\rho_{2} T_{A}^{*}\right)<\frac{\left(C_{B} H^{*}+d_{B}\right)\left(d_{A}+C_{A} H^{*}\right)}{m_{B} C_{B} H^{*}} .
$$

This is important for the following statement.

Theorem 4.1. Assume $1<R_{T}<$ e. Then, the equilibrium $\left(T_{B}^{*}, T_{A}^{*}\right)$ of 4.3 is locally asymptotically stable.

Proof. Since (4.12) holds, (4.13) generates a positive semigroup. Therefore, the stability of (4.13) is equivalent to the stability of the zero solution for the linear ordinary differential equation $16 \mid 17$

$$
\left\{\begin{array}{l}
T_{B}^{\prime}(t)=\rho_{1} \rho_{2} b^{\prime}\left(\rho_{2} T_{A}^{*}\right) T_{A}-\left(C_{B} H^{*}+d_{B}\right) T_{B}, \\
T_{A}^{\prime}(t)=m_{B} C_{B} H^{*} T_{B}-\left(d_{A}+C_{A} H^{*}\right) T_{A},
\end{array}\right.
$$

and the stability of the above solution is determined by whether or not

$$
\left(C_{B} H^{*}+d_{B}\right)\left(d_{A}+C_{A} H^{*}\right)>\rho_{1} \rho_{2} b^{\prime}\left(\rho_{2} T_{A}^{*}\right) m_{B} C_{B} H^{*},
$$

see Corollary 3.2 and the discussion in Ref. 17. In view of (4.14) implied by (4.12), namely $R_{T}<e$, we complete the proof. 
It should also be noted from (4.5) and 4.6) that solutions of 4.3 eventually enter the rectangle $\Pi:=\left\{\left.\left(T_{B}(0), T_{A}\right)\right|_{\left[-\tau_{2}, 0\right]} \mid 0 \leq T_{B}(0) \leq T_{B}^{\infty}\right.$ and $0 \leq T_{A}(\theta) \leq$ $\left.T_{A}^{\infty}, \theta \in\left[-\tau_{2}, 0\right]\right\}$. With $1<R_{T}<e$ and whenever $0 \leq T_{A}^{\epsilon, t} \leq T_{A}^{\infty}, T_{B}(t)=T_{B}^{\infty}$, we have

$$
\begin{aligned}
T_{B}^{\prime}(t) & \leq \rho_{1} b\left(\rho_{2} T_{A}^{\infty}\right)-\left(C_{B} H^{*}+d_{B}\right) T_{B}^{\infty} \\
& =\rho_{1} b\left(\rho_{2} T_{A}^{\infty}\right)-\frac{\left(C_{B} H^{*}+d_{B}\right)\left(d_{A}+C_{A} H^{*}\right)}{m_{B} C_{B} H^{*}} T_{A}^{\infty} \leq 0 .
\end{aligned}
$$

Similarly, whenever $0 \leq T_{B}(t) \leq T_{B}^{\infty}, 0 \leq T_{A}^{\epsilon, t} \leq T_{A}^{\infty}$ and $T_{A}(t)=T_{A}^{\infty}$, we have $T_{A}^{\prime}(t) \leq 0$. Therefore, the set $\Pi$ is also positively invariant, and $(0,0)$ and $\left(T_{B}^{*}, T_{A}^{*}\right)$ are the only equilibria in $\Pi$. Consequently, we also have from the monotone dynamical system theory $\stackrel{16 \mid 17}{ }$ that

Theorem 4.2. If $1<R_{T}<e$, then every nontrivial solution of (4.3) converges to $\left(T_{B}^{*}, T_{A}^{*}\right)$ as $t \rightarrow \infty$.

Note that $R_{T}=R_{T}(C)$ defined in (4.10) is an increasing function of $C>0$, where $C=C_{B} H^{*}$. Therefore, there exists $0<C_{B, 1}<C_{B, 2}$ such that $R_{T}\left(C_{B, 1}\right)=1$ and $R_{T}\left(C_{B, 2}\right)=e$. Therefore, we have shown that system (4.3) has no nontrivial equilibrium if $C<C_{B, 1}$; system (4.3) has a unique positive equilibrium $\left(T_{B}^{*}, T_{A}^{*}\right)$ when $C>C_{B, 1}$; and this positive equilibrium is locally asymptotically stable if $C \in\left(C_{B, 1}, C_{B, 2}\right)$.

The characteristic equation of (4.3) at the given positive equilibrium $\left(T_{B}^{*}, T_{A}^{*}\right)$ is $\operatorname{det}[\lambda I-J]=0$ with the matrix $J$ given by

$$
J=\left(\begin{array}{cc}
-C_{B} H^{*}-d_{B} & J_{12} \\
m_{B} C_{B} H^{*} & -\left(d_{A}+C_{A} H^{*}\right)
\end{array}\right),
$$

with $J_{12}=\alpha_{0}\left[(1-\epsilon) e^{-\lambda \tau_{1}}+\epsilon e^{-\lambda \tau_{2}}\right] e^{-\alpha_{1} T_{A}^{*}}\left(1-\alpha_{1} T_{A}^{*}\right)$. Therefore, the characteristic equation becomes

$$
\begin{aligned}
(\lambda & \left.+C_{B} H^{*}+d_{B}\right)\left(\lambda+d_{A}+C_{A} H^{*}\right)-\alpha_{0} m_{B} C_{B} H^{*}\left(1-\alpha_{1} T_{A}^{*}\right) \\
& \times\left[(1-\epsilon) e^{-\lambda \tau_{1}}+\epsilon e^{\lambda \tau_{2}}\right] e^{-\alpha_{1} T_{A}^{*}}=0
\end{aligned}
$$

That is,

$$
\lambda^{2}+A_{1}\left(C_{B}\right) \lambda+A_{2}\left(C_{B}\right)+A_{3}\left(C_{B}\right) e^{-\lambda \tau_{1}}+A_{4}\left(C_{B}\right) e^{-\lambda \tau_{2}}=0,
$$

where

$$
\begin{aligned}
& A_{1}\left(C_{B}\right)=C_{B} H^{*}+d_{A}+C_{A} H^{*}+d_{B}>0, \\
& A_{2}\left(C_{B}\right)=\left(d_{A}+C_{A} H^{*}\right)\left(C_{B} H^{*}+d_{B}\right)>0, \\
& A_{3}\left(C_{B}\right)=-(1-\epsilon) \alpha_{0} m_{B} C_{B} H^{*}\left(1-\alpha_{1} T_{A}^{*}\right) e^{-\alpha_{1} T_{A}^{*}}, \\
& A_{4}\left(C_{B}\right)=-\epsilon \alpha_{0} m_{B} C_{B} H^{*}\left(1-\alpha_{1} T_{A}^{*}\right) e^{-\alpha_{1} T_{A}^{*}} .
\end{aligned}
$$


Assume that a pair of purely imaginary solution of the form $\lambda= \pm i \omega$ exists in Eq. (4.20), where $\omega$ is a positive real number. Substituting it into (4.20) and separating the real and imaginary parts, we can obtain the following transcendental equations:

$$
\left\{\begin{array}{l}
f_{1}(\omega)=-\omega^{2}+A_{2}\left(C_{B}\right)+A_{3}\left(C_{B}\right) \cos \left(\omega \tau_{1}\right)+A_{4}\left(C_{B}\right) \cos \left(\omega \tau_{2}\right)=0, \\
f_{2}(\omega)=A_{1}\left(C_{B}\right) \omega-A_{3}\left(C_{B}\right) \sin \left(\omega \tau_{1}\right)-A_{4}\left(C_{B}\right) \sin \left(\omega \tau_{2}\right)=0 .
\end{array}\right.
$$

Moving the terms $A_{3}\left(C_{B}\right) \cos \left(\omega \tau_{1}\right)$ and $A_{3}\left(C_{B}\right) \sin \left(\omega \tau_{1}\right)$ into right sides of the equations of (4.21), then taking square on both sides and summing them up, we obtain

$$
\begin{aligned}
\omega^{4} & +\left(A_{1}^{2}-2 A_{2}-2 A_{4} \cos \left(\omega \tau_{2}\right)\right) \omega^{2}-2 A_{1} A_{4} \sin \left(\omega \tau_{2}\right) \omega \\
& +A_{2}^{2}-A_{3}^{2}+A_{4}^{2}+2 A_{2} A_{4} \cos \left(\omega \tau_{2}\right)=0 .
\end{aligned}
$$

Equation 4.22) has a positive real root $\omega^{*}$ if the following condition holds

$$
\left(A_{2}+A_{4}\right)^{2}<A_{3}^{2} .
$$

Since $1-\alpha_{1} T_{A}^{*}<0$ is satisfied, Eq. 4.23 can be reduced into $A_{2}+A_{4}<A_{3}$ due to $A_{3}>0$ and $A_{4}>0$. Therefore, Eq. (4.22) has a positive real root $\omega^{*}$ if

$$
2 \epsilon>1
$$

that is, if at least half of the ticks have gone through the diapause process, and if

$$
C_{B}>\frac{\left(d_{A}+C_{A} H^{*}\right) d_{B}}{H^{*}}\left(\alpha_{0} m_{B} e^{-1+\frac{1}{2 \epsilon-1}}-d_{A}-C_{A} H^{*}\right)^{-1} .
$$

In what follows, we define $C_{B}^{*}$ as the minimal contact rate (larger than $C_{B, 2}$ ) at which there is a pair of purely imaginary eigenvalues (denoted by $\pm i \omega^{*}$ ). To confirm there is indeed a Hopf bifurcation of periodic solutions bifurcated from the equilibrium, we need to verify the transversal condition. Differentiating (4.20) with respect to $C_{B}$ and substituting the eigenvalue $\omega^{*}$ and critical value $C_{B}^{*}$, we obtain

$$
\begin{array}{r}
\left.P\left(\frac{d(\operatorname{Re} \lambda)}{d C_{B}}\right)\right|_{C_{B}^{*}}+\left.Q\left(\frac{d \omega}{d C_{B}}\right)\right|_{C_{B}^{*}}=R, \\
-\left.Q\left(\frac{d(\operatorname{Re} \lambda)}{d C_{B}}\right)\right|_{C_{B}^{*}}+\left.P\left(\frac{d \omega}{d C_{B}}\right)\right|_{C_{B}^{*}}=S,
\end{array}
$$

where

$$
\begin{aligned}
& P=A_{1}\left(C_{B}\right)-\tau_{1} A_{3}\left(C_{B}\right) \cos \left(\omega \tau_{1}\right)-\tau_{2} A_{4}\left(C_{B}\right) \cos \left(\omega \tau_{2}\right), \\
& Q=-2 \omega-\tau_{1} A_{3}\left(C_{B}\right) \sin \left(\omega \tau_{1}\right)-\tau_{2} A_{4}\left(C_{B}\right) \sin \left(\omega \tau_{2}\right), \\
& R=-A_{2}^{\prime}\left(C_{B}\right)-A_{3}^{\prime}\left(C_{B}\right) \cos \left(\omega \tau_{1}\right)-A_{4}^{\prime}\left(C_{B}\right) \cos \left(\omega \tau_{2}\right), \\
& S=-A_{1}^{\prime}\left(C_{B}\right) \omega+A_{3}^{\prime}\left(C_{B}\right) \sin \left(\omega \tau_{1}\right)+A_{4}^{\prime}\left(C_{B}\right) \sin \left(\omega \tau_{2}\right) .
\end{aligned}
$$


From these equations, we can have the transversality condition

$$
\left.\left(\frac{d(\operatorname{Re} \lambda)}{d \tau_{1}}\right)\right|_{C_{B}^{*}}:=\frac{P R-Q S}{P^{2}+Q^{2}} \neq 0 .
$$

Therefore, assuming that the condition (4.25) holds and (4.27) is nonzero, then the positive equilibrium $E$ of model (3.6) is locally asymptotically stable for $C_{B} \in$ $\left(C_{B, 1}, C_{B}^{*}\right)$ and there undergoes a Hopf bifurcation at $C_{B}=C_{B}^{*}$.

To summarize, we have

Theorem 4.3. Assume $R_{T}>e, 2 \epsilon>1$. Then, there exists $C_{B}^{*}>C_{B, 2}$ so that at $C_{B}^{*}$, the linearization at the nontrivial equilibrium $\left(T_{B}^{*}, T_{A}^{*}\right)$ has a pair of purely imaginary eigenvalues $\pm i \omega^{*}$ with $\omega^{*}>0$. Assuming the transversality condition (4.27) is satisfied, then the model system (3.6) undergoes a Hopf bifurcation.

In Sec. 6. we will numerically examine an example to calculate the corresponding critical contact rate $C_{B}$ for such a Hopf bifurcation to take place.

\section{Implication for Pathogen Spread}

Substituting constant solutions $T_{B}^{*}$ and $T_{A}^{*}$ into the full epidemic model (3.1)-(3.8), we now consider the following system relevant to infected ticks and hosts:

$$
\begin{aligned}
T_{A i}^{\prime}(t)= & \eta_{c} m_{B} C_{B} T_{B}^{*} H_{s^{+}}+\left(\eta_{s}+\eta_{c}-\eta_{s} \eta_{c}\right) m_{B} C_{B} T_{B}^{*} H_{i^{+}} \\
& +\eta_{s} m_{B} C_{B} T_{B}^{*} H_{i^{-}}-C_{A} T_{A i} H^{*}-d_{A} T_{A i}, \\
H_{s^{+}}^{\prime}(t)= & \left(1-\eta_{h}\right) C_{A}\left(H^{*}-H_{s^{+}}-H_{i^{+}}-H_{i^{-}}\right) T_{A i} \\
& -\eta_{h} C_{A} H_{s^{+}} T_{A i}-\left(d_{f}+d_{h}\right) H_{s^{+}}, \\
H_{i^{+}}^{\prime}(t)= & \eta_{h} C_{A} H_{s^{+}} T_{A i}+\eta_{h} C_{A}\left(H^{*}-H_{s^{+}}-H_{i^{+}}-H_{i^{-}}\right) T_{A i} \\
& +C_{A} H_{i^{-}} T_{A i}-\left(d_{f}+d_{h}\right) H_{i^{+}}, \\
H_{i^{-}}^{\prime}(t)= & -C_{A} H_{i^{-}} T_{A i}+d_{f} H_{i^{+}}-d_{h} H_{i^{-}} .
\end{aligned}
$$

We can define and calculate the basic reproduction ratio of pathogen population of system (5.1) following the idea in Ref. 18 In particular, we linearize system (5.1) at the disease-free equilibrium $\left(T_{A i}, H_{s^{+}}, H_{i^{+}}, H_{s^{-}}\right)=(0,0,0,0)$ to obtain the new infection matrix

$$
F=\left(\begin{array}{cccc}
0 & \eta_{c} m_{B} C_{B} T_{B}^{*} & \left(\eta_{s}+\eta_{c}-\eta_{s} \eta_{c}\right) m_{B} C_{B} T_{B}^{*} & \eta_{s} m_{B} C_{B} T_{B}^{*} \\
\left(1-\eta_{h}\right) C_{A} H^{*} & 0 & 0 & 0 \\
\eta_{h} C_{A} H^{*} & 0 & 0 & 0 \\
0 & 0 & 0 & 0
\end{array}\right)
$$

and the progression matrix

$$
V=\left(\begin{array}{cccc}
C_{A} H^{*}+d_{A} & 0 & 0 & 0 \\
0 & d_{f}+d_{h} & 0 & 0 \\
0 & 0 & d_{f}+d_{h} & 0 \\
0 & 0 & -d_{f} & d_{h}
\end{array}\right) .
$$


Thus, the basic reproduction ratio $\mathcal{R}_{0}$ of pathogen population of system (5.1) is the spectral radius of matrix $F V^{-1}$, given by

$$
\begin{aligned}
\mathcal{R}_{0} & =\rho\left(F V^{-1}\right) \\
& =\sqrt{\frac{C_{A} H^{*} m_{B} C_{B} T_{B}^{*}}{C_{A} H^{*}+d_{A}}\left(\frac{\left(1-\eta_{h}\right) \eta_{c}}{d_{f}+d_{h}}+\frac{\eta_{h}\left(\eta_{s}+\eta_{c}-\eta_{s} \eta_{c}\right)}{d_{f}+d_{h}}+\frac{\eta_{h} \eta_{s}}{d_{h}} \frac{d_{f}}{d_{f}+d_{h}}\right) .}
\end{aligned}
$$

Furthermore, the value of $\mathcal{R}_{0}$ defined in Eq. (5.2) can offer as a threshold to characterize the co-existence of pathogen population in the following theorem.

Theorem 5.1. The following statements are valid:

(ii) If $\mathcal{R}_{0}<1$, system (5.1) admits a unique zero equilibrium;

(ii) If $\mathcal{R}_{0}>1$, system (5.1) admits a unique positive equilibrium $E_{1}=\left(T_{A i}^{*}, H_{s^{+}}^{*}, H_{i^{+}}^{*}, H_{i^{-}}^{*}\right)$.

Proof. Let

$$
x_{1}=T_{A i}(t), \quad x_{2}=H_{s^{+}}(t), \quad x_{3}=H_{i^{+}}(t), \quad x_{4}=H_{i^{-}}(t) .
$$

Assuming all right hands of equations of system (5.1) be zeros, and then adding the second and third equations and third and fourth equations, respectively, lead to

$$
x_{2}+x_{3}=\frac{C_{A} H^{*} x_{1}}{C_{A} x_{1}+d_{f}+d_{h}} \quad \text { and } \quad x_{3}+x_{4}=\frac{\eta_{h} C_{A} H^{*} x_{1}}{\eta_{h} C_{A} x_{1}+d_{h}} .
$$

Moreover, from the fourth equation of system (5.1), we obtain

$$
x_{3}=\frac{C_{A} x_{1}+d_{h}}{d_{f}} x_{4} .
$$

Thus, through substituting Eq. (5.5) into above Eqs. (5.4), we can express the expressions of $x_{2}, x_{3}$ and $x_{4}$ in terms of $x_{1}$ as:

$$
\begin{aligned}
& x_{4}=\frac{\eta_{h} C_{A} x_{1} H^{*}}{\eta_{h} C_{A} x_{1}+d_{h}} \frac{d_{f}}{C_{A} x_{1}+d_{f}+d_{h}}, \\
& x_{3}=\frac{\eta_{h} C_{A} x_{1} H^{*}}{\eta_{h} C_{A} x_{1}+d_{h}} \frac{C_{A} x_{1}+d_{h}}{C_{A} x_{1}+d_{f}+d_{h}}, \\
& x_{2}=\frac{C_{A} x_{1} H^{*}}{C_{A} x_{1}+d_{f}+d_{h}} \frac{\left(1-\eta_{h}\right) d_{h}}{\eta_{h} C_{A} x_{1}+d_{h}} .
\end{aligned}
$$

Substituting the above expressions of $x_{2}, x_{3}$ and $x_{4}$ into the right hand of the first equation from system (5.1) and letting this be zero, we can obtain a quadratic equation with respect to nonzero variable $x_{1}$ as follows:

$$
a x_{1}^{2}+b x_{1}+c=0
$$


where

$$
\begin{aligned}
a= & \left(C_{A} H^{*}+d_{A}\right) \eta_{h} C_{A}^{2}, \\
b= & \left(C_{A} H^{*}+d_{A}\right) C_{A}\left(d_{h}+\eta_{h}\left(d_{f}+d_{h}\right)\right)-m_{B} C_{B} T_{B}^{*} C_{A}^{2} H^{*}\left(\eta_{s}+\eta_{c}-\eta_{s} \eta_{c}\right) \eta_{h}, \\
c= & \left(C_{A} H^{*}+d_{A}\right) d_{h}\left(d_{f}+d_{h}\right)-m_{B} C_{B} T_{B}^{*} C_{A} H^{*}\left(\left(1-\eta_{h}\right) \eta_{c} d_{h}\right. \\
& \left.+\eta_{h}\left(\eta_{s}+\eta_{c}-\eta_{s} \eta_{c}\right) d_{h}+\eta_{h} \eta_{s} d_{f}\right) \\
= & \left(C_{A} H^{*}+d_{A}\right) d_{h}\left(d_{f}+d_{h}\right)\left(1-\mathcal{R}_{0}^{2}\right) .
\end{aligned}
$$

Denoting by $\lambda_{1}$ and $\lambda_{2}$, the two solutions of the above quadratic equation (5.9), we obtain

$$
\lambda_{1}+\lambda_{2}=\frac{d_{h}+\eta_{h}\left(d_{f}+d_{h}\right)}{\eta_{h} C_{A}}\left(\frac{m_{B} C_{B} T_{B}^{*} C_{A} H^{*}}{C_{A} H^{*}+d_{A}} \frac{\eta_{h}\left(\eta_{s}+\eta_{c}-\eta_{s} \eta_{c}\right)}{d_{h}+\eta_{h}\left(d_{f}+d_{h}\right)}-1\right) .
$$

One can prove that

$$
\frac{\eta_{h}\left(\eta_{s}+\eta_{c}-\eta_{s} \eta_{c}\right)}{d_{h}+\eta_{h}\left(d_{f}+d_{h}\right)}<\frac{\left(1-\eta_{h}\right) \eta_{c}}{d_{f}+d_{h}}+\frac{\eta_{h}\left(\eta_{s}+\eta_{c}-\eta_{s} \eta_{c}\right)}{d_{f}+d_{h}}+\frac{\eta_{h} \eta_{s}}{d_{h}} \frac{d_{f}}{d_{f}+d_{h}} .
$$

Accordingly, we have

$$
\lambda_{1}+\lambda_{2}<\frac{d_{h}+\eta_{h}\left(d_{f}+d_{h}\right)}{\eta_{h} C_{A}}\left(\mathcal{R}_{0}^{2}-1\right)
$$

and

$$
\lambda_{1} \lambda_{2}=\frac{d_{h}\left(d_{f}+d_{h}\right)}{\eta_{h} C_{A}^{2}}\left(1-\mathcal{R}_{0}^{2}\right) .
$$

Obviously, when $\mathcal{R}_{0}>1$, there exists a unique positive solution $x_{1}^{*}>0$ of Eq. (5.9); when $\mathcal{R}_{0}<1$, there is no positive solution of $x_{1}$ of Eq. (5.9). When there is a unique positive solution of $x_{1}^{*}$, we can easily find the unique positive solution of $x_{2}, x_{3}$ and $x_{4}$ of system (5.1). This completes the proof.

We also note that when $C_{B}$ crosses the first bifurcation value $C_{B}^{*}$, the tick system (3.6) admits a unique periodic solution of $T_{B}^{*}(t)$ and $T_{A}^{*}(t)$. Substituting $T_{B}^{*}(t)$ and $T_{A}^{*}(t)$ into the full system, we can have a limiting periodic system and we can then follow the procedure in Refs. [3] and 19] to define a next generation operator and its spectral radius as the basic reproduction number which is close to the formula defined in Eq. (5.2) where $C_{B}$ is close to $C_{B}^{*}$ due to the continuity of the spectral radius.

\section{Numerical Illustrations}

We use some numerical simulations to illustrate the theoretical stability and bifurcation analysis. This sets a stage for our final discussions about the possible research topics in the future. 
We mention that for the purpose of numerical simulations, all parameter values listed below are purely illustrative, though elaborated from the literature $6 \sqrt{20 \mid 21}$ We fix $\rho_{1}=0.0252, \rho_{2}=0.1, r_{T}=2000\left(\right.$ day $\left.^{-1}\right), s_{T}=0.001, \tau_{1}=750$ (day), $\tau_{2}=1100$ (day), $\epsilon=0.6, d_{B}=0.0277\left(\right.$ day $\left.^{-1}\right), d_{A}=0.0277\left(\right.$ day $\left.^{-1}\right), m_{B}=1$, $\eta_{c}=0.0004, \eta_{s}=0.8, C_{A}=0.001, \eta_{h}=0.8, d_{f}=0.2\left(\right.$ day $\left.^{-1}\right), d_{h}=0.087\left(\right.$ day $\left.^{-1}\right)$, $r_{h}=0.1\left(\right.$ day $\left.^{-1}\right), K_{h}=200\left(\right.$ day $\left.^{-1}\right)$.

A stability and bifurcation analysis for even a scalar delay differential equation with two distinct delays is always difficult. In most existing analyses, the two time lags are considered as bifurcation parameters. In the model system, we are considering the biological issues relevant to diapause and co-feeding suggest two other parameters: the parameter $\epsilon$, the portion when the population has to go through a diapause process to gain maturity after time lag $\tau_{2}$ and the contact rate $C_{B}$ for the development of ticks to the next stage before co-feeding. In our case, motivated by tick-borne encephalitis and Lyme disease, $\tau_{1}$ and $\tau_{2}$ are relatively fixed to approximately 2 and 4-years. Therefore, $\tau_{1}$ as 750 (days) and $\tau_{2}$ as 1100 (days) and $\epsilon=0.6$ so that $2 \epsilon>1$.

When $C_{B}=0.7 \times 10^{-4}\left(\right.$ day $\left.^{-1}\right)$, there exists a positive equilibrium (485440, 18803, 26) for the model (3.6), but the algebraic equation (4.22) has no positive solution, leading to positive steady state solution of tick population which is locally asymptotically stable. Moreover, we can calculate the basic reproduction ratio of pathogen population as 11.0017 which is greater than 1 . Thus, by Theorem 5.1 the epidemic model (5.1) admits a positive steady state $(13182,0.3254,21.0260,2.9926)$ which is locally asymptotically stable as shown in Fig. 3(a).

Based on the theoretical analysis, we can calculate the critical value of the contact rate $C_{B}^{*}=1.195 \times 10^{-4}\left(\right.$ day $\left.^{-1}\right)$, this leads to the corresponding positive equilibrium $E(388480,22477,26)$ of the model (3.6), and Eq. (4.21) has a unique

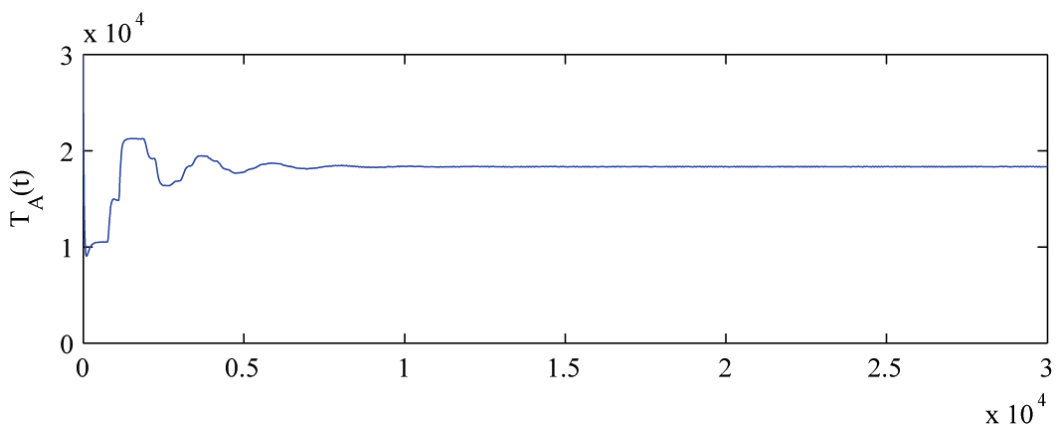

(a)

Fig. 3. Solutions of $T_{A}(t)$ for different contact rate (a) $C_{B}=0.7 \times 10^{-4}$, (b) $C_{B}=1.25 \times 10^{-4}$; (c) $C_{B}=2.5 \times 10^{-4}$, and (d) $C_{B}=3.3 \times 10^{-4}$. (a) $T_{A}(t)$ approaches a positive steady state; (b) $T_{A}(t)$ approaches a periodically oscillatory solution; (c) solution of $T_{A}(t)$ approaches a dualpeak periodic function when $C_{B}$ is increased to $2.5 \times 10^{-4}$; (d) solution of $T_{A}(t)$ increases the complexity of oscillation when $C_{B}$ is further increased to $3.3 \times 10^{-4}$. 


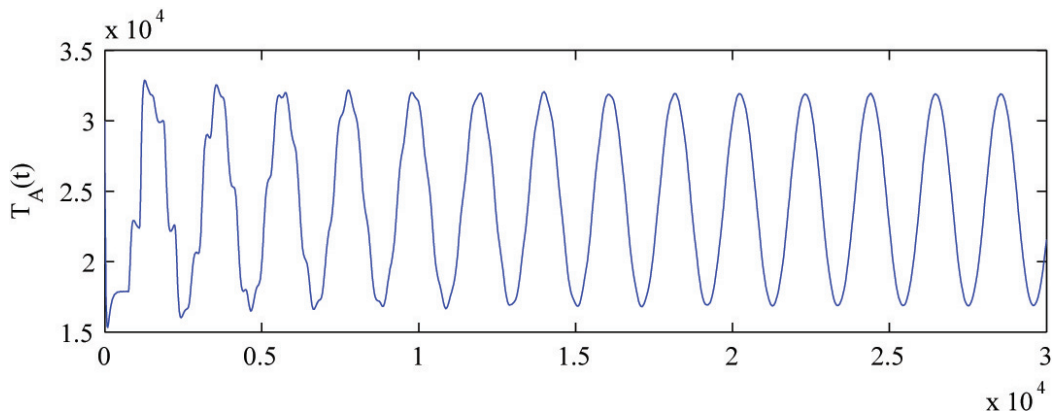

(b)

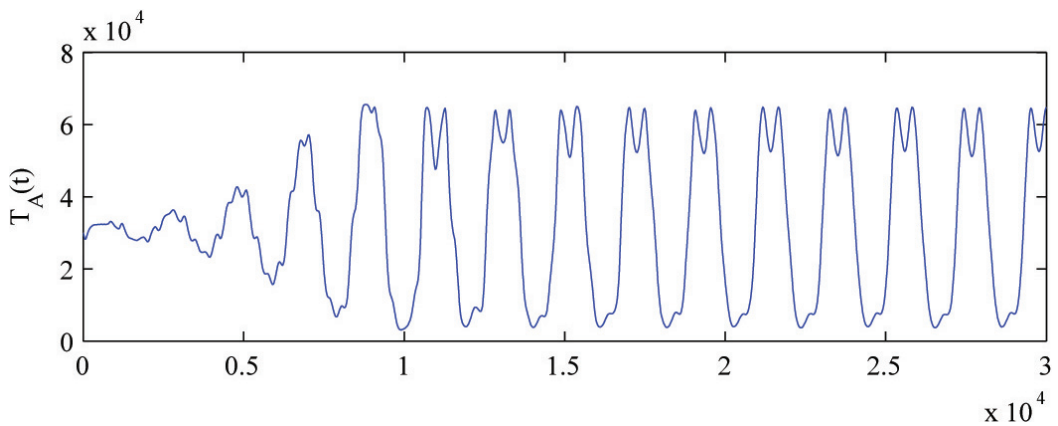

(c)

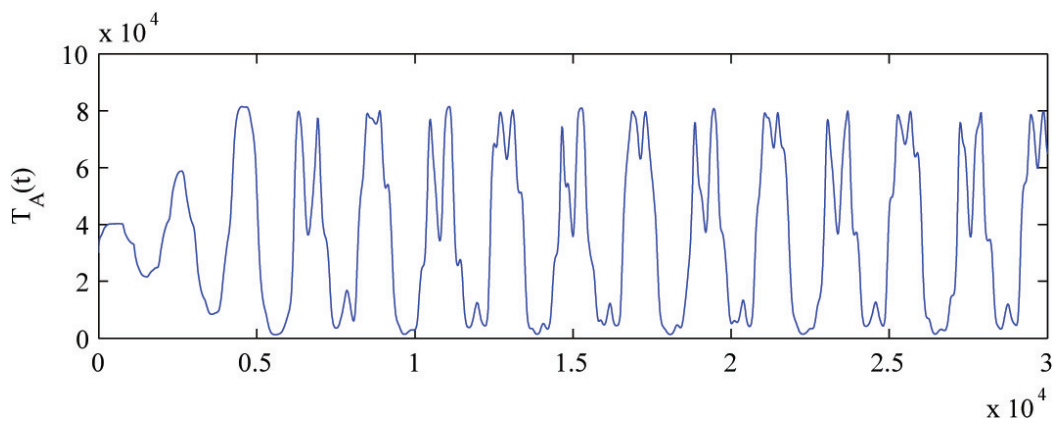

(d)

Fig. 3. (Continued)

solution $\omega^{*}=3.4199 \times 10^{-3}$ corresponding to this $C_{B}^{*}$. Thereby, according to the Hopf Bifurcation Theorem, model (3.6) undergoes a Hopf bifurcation at $E$ and a bifurcated periodic solution exists for $C_{B}$ slightly larger than $C_{B}^{*}$, as depicted in Fig. 3(b). The period is 2100 (days), close to double the size of $\tau_{2}$.

Increasing the contact rate further generates multiple peaks and valleys in both tick population and infections. For instance, when we choose $C_{B}=2.5 \times 10^{-4}$, 
within the period of a periodic solution, there are dual-peaks in both tick population and infections, where the period is also increased to 2310 days (see Fig. [3](c)). Increasing the contact rate further to $3.3 \times 10^{-4}$, we observe more complex oscillations in the infected A-stage ticks while the period is also increased (see Fig. 33(d)).

\section{Discussions}

In our theoretical analyses and simulations, we have used the contact rate $C_{B}$ as the bifurcation parameter to examine the impact of diapause on nonlinear oscillations when co-feeding takes place. We have fixed the parameter $\epsilon$, the fraction of ticks going through the diapause process to reach maturity with some delays. It will be a very interesting problem for future study to conduct the bifurcation analysis when we change $\epsilon$, and in particular, to look at the critical diapause fraction (when two delays $\tau_{1}$ and $\tau_{2}$ are fixed) when Hopf bifurcation takes place. A good starting point would be to consider the case where the tick population model exhibits convergence to equilibria without diapause, and exhibits convergence to periodic solutions when all populations have to go through the diapause to reach the maturity.

Annual periodic cycles of tick population dynamics have been frequently observed in the fields and theoretically confirmed in the model study ${ }^{22}$ where a system of ordinary differential equations is used and where periodic coefficients are assumed to represent the seasonal variation of tick ecological activities including questing, feeding, development, reproduction and mortality. These annual cycles have also been established in the work, ${ }^{23}$ using a system of delay differential equations with both periodic delays (for development times) and periodic coefficients. When these periodic coefficients and delays are replaced by constants, the model systems converge to equilibrium states. Therefore, the periodicity observed in these studies is caused by the seasonal variations and the period of the periodic solutions is fixed to one year. Here, we show that there is another mechanism for nonlinear oscillations in tick population dynamics, arising from diapause which is caused by environmental conditions to delay the development of ticks from one stage to the next by a year or two. These diapause-caused oscillations are generally not in synchrony with the seasonality, and multiple peaks and valleys can appear within a single period. While the oscillation frequency of the diapause-induced periodic oscillations can be estimated using model parameters, from the bifurcation analysis (at least when the bifurcation value $C_{B}$ is close to the critical value), the distance between consequent peaks within a single period is hard to estimate. Developing effective methods to estimate this distance remains to be a topics for future research and this will be practically useful for predicting the (temporally) local outbreak.

Our model formulation is motivated by the co-feeding transmission observed in tick-borne diseases, and for the sake of simplicity, we have chosen our focus on the transition of the ticks between the two stages relevant to the co-feeding. As such, we have ignored a few physiologically distinct stages and the model parameters, specially the development delay and the development and survival rates, all involve 
distinct stages for which human interventions and environmental conditions have different effects. To fit the model with real surveillance data and to use the model to evaluate the impact of human interventions and environmental variation on controlling tick population growth and tick-borne disease spread, we need to refine the model to incorporate all four different tick development stages and also to use periodic coefficients rather than constant coefficients to reflect the seasonal variation aforementioned. The diapause-induced oscillation and the seasonal variations combined are expected to generate more complicate tick population dynamics oscillations, which in turn will generate complex temporal patterns of tick-borne disease dynamics.

\section{Acknowledgments}

This research has been supported by the Natural Sciences and Engineering Research Council of Canada and the Canada Research Chairs Program. Authors also appreciate the financial support of NSFC (Nos. 11501358 and 11701072) and FRQNT. Correspondence should be sent to Xue Zhang (zhangxue@mail. neu.edu.cn) and/or Xiaotian Wu (xtwu@shmtu.edu.cn).

\section{References}

1. Rizzoli A, Hauffe H, Tagliapietra V, Neterler M, Roberto R, Forest structure and roe deer abundance predict tickborne encephalitis risk in Italy, PLoS One 4:e4336, 2009.

2. Kiffner C, Zucchini W, Schomaker P, Vor T, Hagedorn P, Niedrig M, Rhe F, Determinants of tick-borne encephalitis in counties of southern Germany, Int J Health Geogr 9:2001-2008, 2010.

3. Bacaër N, Guernaoui S, The epidemic threshold of vector-borne diseases with seasonality: the case of cutaneous leishmaniasis in Chichaoua, Morocco, J Math Biol 53:421-436, 2006.

4. Foley P, Foley J, Modeling susceptible infective recovered dynamics and plague persistence in California rodent-flea communities, Vector Borne Zoonotic Dis 10:59-67, 2010 .

5. Marquardt WC, Biology of Disease Vectors, 2nd edn., Elservier Academic Press, USA, 2005.

6. Rosà R, Pugliese A, Effects of tick population dynamics and host densities on the persistence of tick-borne infections, Math Biosci 208:216-240, 2007.

7. Dunn JM, Davis S, Stacey A, Diuk-Wasser MA, A simple model for the establish of tick-borne pathogens of Ixodes scapularis: A global sensitivity analysis of $R_{0}, J$ Theor Biol 335:213-221, 2013.

8. Lou Y, Wu J, Wu X, Impact of biodiversity and seasonality on Lyme-pathogen transmission, Theor Biol Med Model 11:50, 2014.

9. Dennis D, Piesman J, Overview of tick-borne infections of humans, in Goodman J, Dennis D, Sonenshine D (eds.), Tick-borne Diseases of Humans, ASM Press, Washington, DC, pp. 3-11, 2005.

10. Labuda M, Kozuch O, Zuffova E, Eleckova E, Hails RS, and Nuttall PA, Tick-borne encephalitis virus transmission between ticks cofeeding on specific immune natural rodent hosts, Virology 235:138-143, 1997. 
11. Voordouw MJ, Co-feeding transmission in Lyme disease pathogens, Parasitology 142:290-302, 2015.

12. Graya JS, Kahlb O, Lanec RS, Levind ML, Tsaoe JI, Diapause in ticks of the medically important Ixodes ricinus species complex, Ticks Tick Borne Dis 7(5):992-1003, 2016.

13. Madder M, Speybroeck N, Brandt J, Tirry L, Hodek I, Berkvens D, Geographic variation in diapause response of adult Rhipicephalus appendiculatus ticks, Exp Appl Acarol 27(3):209-221, 2002.

14. Belozerov VN, Naumov RL, Nymphal diapause and its photoperiodic control in the tick Ixodes scapularis (Acari: Ixodidae), Folia Parasitol 49:314-318, 2002.

15. Ricker WE, Computation and Interpretation of Biological Statistics of Fish Populations, Bull. Fish. Res. Board Can. No. 191, Blackburn Press, Ottawa, 1975.

16. Smith H, Monotone Dynamical Systems, an Introduction to the Theory of Competitive and Cooperative Systems, American Mathematical Society, Mathematical Surveys and Monographs, 1995.

17. Smith H, Monotone semiflows generated by functional differential equations, $J$ Differ Eq 87:420-442, 1987.

18. van den Driessche P, Watmough J, Reproduction numbers and sub-threshold endemic equilibria for compartmental models of disease transmission, Math Biosci 180:29-48, 2002.

19. Wang W, Zhao X-Q, Threshold dynamics for compartmental epidemic models in periodic environments, J Dyn Diff Eq 20:699-717, 2008.

20. Rosá R, Pugliese A, Norman R, Hudson PJ, Thresholds for disease persistence in models for tick-borne infections including non-viraemic transmission, extended feeding and tick aggregation, $J$ Theor Biol 224:359-376, 2003.

21. Hartemink NA, Randolph SE, Davis SA, Heesterbeek JAP, The basic reproduction number for complex disease systems: defining $R_{0}$ for tick-borne infections, Am Nat 171(6):743-754, 2008.

22. Wu X, Duvvuri V, Lou Y, Ogden N, Pelcat Y, Wu J, Developing a temperature-driven map of the basic reproductive number of the emerging tick vector of Lyme disease Ixodes scapularis in Canada, J Theor Biol 319:50-61, 2013.

23. Wu X, Magpantay F, Wu J, Zou X, Stage-structured population systems with temporally periodic delay, Math Meth Appl Sci 38(16):3464-3481, 2015. 